\title{
TME Quality in Rectal CANCER Surgery
}

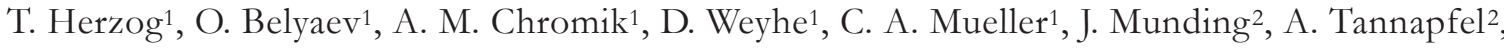 \\ W. Uhl ${ }^{1}$, M. H. Seelig ${ }^{1}$

\footnotetext{
${ }^{1}$ Department of Surgery, St. Josef Hospital, Ruhr-University Bochum, School of Medicine, Germany, ${ }^{2}$ Department of Pathology, BG- Hospital Bergmannsheil, Ruhr- University Bochum, School of Medicine, Germany
}

\begin{abstract}
Background: The concept of total mesorectal excision has revolutionised rectal cancer surgery. TME reduces the rate of local recurrence and tumour associated mortality. However, in clinical trials only $50 \%$ of the removed rectal tumours have an optimal TME quality. Patients: During a period of 36 months we performed 103 rectal resections. The majority of patients $(76 \%$; $78 / 103)$ received an anterior resection. The remaining patients underwent either abdominoperineal resection $(16 \% ; 17 / 103)$, Hartmann`s procedure $(6 \% ; 6 / 103)$ or colectomy $(2 \% ; 2 / 103)$.

Results: In 90\% (93/103) TME quality control could be performed. 99\% (92/93) of resected tumours had optimal TME quality. In 1\% (1/93) the mesorectum was nearly complete. None of the removed tumours had an incomplete mesorectum. In 98\% (91/93) the circumferential resection margin was negative. Major surgical complications occurred in 17\% (18/103). 5\% $(4 / 78)$ of patients with anterior resection had anastomotic leakage. $17 \%(17 / 103)$ developed wound infections. Mortality after elective surgery was 4\% (4/95). Conclusion: Optimal TME quality results can be achieved in all stages of rectal cancer with a rate of morbidity and mortality comparable to the results from the literature. Future studies should evaluate outcome and local recurrence in accordance to the degree of TME quality.
\end{abstract}

Key words: rectal cancer, surgery, total mesorectal excision

\section{BACKGROUND}

The concept of total mesorectal excision (TME) has been the most important development in rectal cancer surgery during the last two decades. After the introduction of TME the rate of local recurrence could be dramatically reduced [1]. Even without curative approach, local recurrence was reduced to $6-12 \%$ and 5 year survival rate improved to $53-87 \%$ [2-4]. However, it is noteworthy, that the excellent results of a local recurrence rate of less than $5 \%$ without neoadjuvant treatment modalities as reported by Heald have not been reached by the majority of rectal surgeons [1].

TME quality is often referred to the graduation of the M.E.R.C.U.R.Y. study that was based on the classification of malignant gastrointestinal tumours [5].
The protocol differentiates between three levels of surgical quality ranging from complete (M.E.R.C.U.R.Y. I. $^{\circ}$ ) to incomplete TME (M.E.R.C.U.R.Y. III. ${ }^{\circ}$ ) (Table 1).

A recent multicentre trial analysed the benefit of preoperative radiation before TME surgery. Surgeons were trained in TME surgery before participating in the study. Nevertheless only $50 \%$ of resected specimens had an optimal TME quality [6, 7]. If optimal TME-quality could be achieved in a controlled scientific trial in only $50 \%$ of patients, serious concern should arise about the TME quality in the absence of pathological quality control.

Although most centres claim performing TME surgery, the literature evaluating TME quality is scarce. To close this gap we present our results of TME surgery after the introduction of quality controls for rectal cancer surgery at our centre in 2004.

\section{Patients and Methods}

During a period of 36 months, between January 2004 and December 2006, 103 patients underwent surgical resection for rectal cancer at the Department of Surgery, St. Josef Hospital, Ruhr- University Bochum, Germany. Sixty percent $(62 / 103)$ were male, $40 \%$ $(41 / 103)$ were female. Mean age was 68.5 years, with a range from 38 to 95 years.

Preoperative staging included complete colonoscopy or barium enema, abdominal CT scan and chest $\mathrm{X}$-ray. Endorectal ultrasound was performed in $76 \%$ (78/103) of patients. MRI was not routinely performed. The decision about neoadjuvant therapy was based on weekly multidisciplinary tumour board reviews. In $29 \%$ (30/103) neoadjuvant therapy was performed prior to operation, including $22 \%(23 / 103)$ short term radiation (5x5 Gy) and 7\% (7/103) long term chemoradiation (50 Gy). In-hospital death was defined as death within 30 postoperative days.

\section{TME SURGERY}

All patients underwent surgery according to the principles of TME. After anterior resection, reconstruction was achieved via stapled anastomosis $(29 \mathrm{~mm}$ or $31 \mathrm{~mm}$ stapler) or hand sutured coloanal anastomosis. Reconstruction included the formation of a colonic pouch by performing a transverse coloplasty whenever possible [8]. 
Table 1. Quality of mesorectal excision according to M.E.R.C.U.R.Y. criteria [5].

\begin{tabular}{llll}
\hline M.E.R.C.U.R.Y. I & Complete & Mesorectum & Smooth, intact \\
& & Defects & Not deeper than $5 \mathrm{~mm}$ \\
& & Coning & No coning \\
& CRM & Smooth, regular \\
\hline M.E.R.C.U.R.Y. II & \multirow{2}{*}{ Nearly complete } & Mesorectum & Moderate bulk, irregular \\
& & Defects & No visible muscularis propria \\
& & Coning & Moderate \\
& & CRM & Irregular \\
\hline M.E.R.C.U.R.Y. III & Incomplete & Mesorectum & Little bulk \\
& & Defects & Down onto muscularis propria \\
& & Coning & Yes \\
& & CRM & Irregular \\
\hline
\end{tabular}

Table 2. Major complications after surgery for rectal cancer.

\begin{tabular}{llrr}
\hline Surgical complications & Anastomotic leakage & 4 & $5 \%$ \\
$(\mathrm{n}=18)$ & Wound dehiscence & 4 & $4 \%$ \\
& Intraabdominal abscess & 4 & $4 \%$ \\
& Bleeding & 2 & $2 \%$ \\
& Hartmann`s stump leakage & 3 & $50 \%$ \\
& Ureter injury & 1 & $1 \%$ \\
\hline General complications & Pneumonia & 5 & $5 \%$ \\
$(\mathrm{n}=7)$ & Stroke & 1 & $1 \%$ \\
& Myocardial infarction & 1 & $1 \%$ \\
\hline
\end{tabular}

\section{TME QUALITY}

Histopathological examination was performed according to the protocol of Quirke et al. [9]. The quality of the surgical resections was first judged by macroscopic assessment of the specimens' surface. After fixation, staining and slicing, the completeness of the mesorectum was judged by microscopic investigation. Tumour type, T-Stage and N-Stage were documented for the purposes of the study. Further recording included proximal, distal and circumferential resection margins, tumour size and histopathological grading. The quality of the mesorectum was then determined according to the M.E.R.C.U.R.Y. study guidelines by two different pathologists (Table 1).

\section{RESULTS}

\section{Operative Results}

Ninetynine percent $(102 / 103)$ of the removed tumours were adenocarcionomas of the rectum. One patient with malignant melanoma of the anus had abdominoperineal resection. $8 \%(8 / 103)$ of patients needed urgent surgery secondary to ileus $(3 \% ; 3 / 103)$ or perforation of rectal carcinoma $(5 \% ; 5 / 103)$. In $76 \%(78 / 103)$ an anterior resection was performed, $6 \%$ underwent $(6 / 103)$ Hartmann`s operation and $2 \%(2 / 103)$ had a colectomy. In $16 \%(17 / 103)$ of cases an abdominoperineal resection was performed. These patients either had local recurrence of rectal cancer, a tumour less than $2 \mathrm{~cm}$ from the anal verge, sphincter infiltration, sphincter insufficiency or malignant melanoma of the anus.

Major surgical complications occurred in $17 \%$ $(18 / 103)$ of patients, including anastomotic leakage, wound dehiscence, intra-abdominal abscess formation, postoperative haemorrhage and failure of the rectal remnant after Hartmann' operation. Major general complications occurred in 7\% (7/103) including pneumonia, stroke and myocardial infarction (Table 2). Mortality after elective operations was 4\% (4/95), while patients undergoing urgent surgery had a mortality rate of $38 \%(3 / 8), p=0.026$.

\section{Histopathological Results}

In $90 \%(93 / 103)$ an evaluation of the quality of the mesorectum was possible. In $10 \%(10 / 103)$ quality analysis was impossible. 7 patients had local recurrence of rectal cancer and had already had rectal resection during prior surgery. 3 patients were part of another study which required immediate opening of the removed rectum for the collection of unfixed tumour biopsies from the removed tumour.

The analysis showed a complete mesorectum (M.E.R.C.U.R.Y. I ${ }^{\circ}$ ) in $99 \%(92 / 93)$ of removed tumours. In 1\% (1/93) the mesorectum was nearly complete (M.E.R.C.U.R.Y. $\left.\mathrm{II}^{\circ}\right)$, and none $(0 / 93)$ of the removed tumours had an incomplete mesorectum (M.E.R.C.U.R.Y. $\mathrm{III}^{\circ}$ ). Macroscopic TME quality is shown in Figure 1.

In $98 \%(91 / 93)$ the circumferential resection margin was negative, showing no tumour infiltration at least 2 millimeters away from the lateral resection margin.

One patient requiring urgent surgery for perforated rectal cancer (UICC tumour stage IIIc) had involvement of the lateral resection margin. The other patient with lateral resection margin involvement was operated after long term chemoradiation (UICC tumour stage IIIb). 


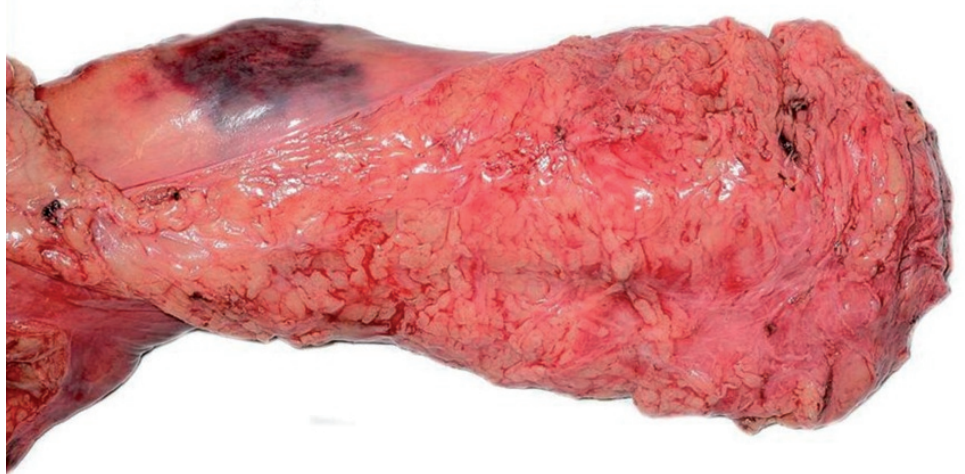

Fig. 1. Macroscopic high TME quality of an unfixed rectal resection specimen showing the intact mesorectum without defects on the surface. The CRM is regular and smooth.
Table 3. UICC tumour stage; final histology $(\mathrm{n}=103)$.

\begin{tabular}{lcc}
\hline \multicolumn{3}{l}{ Histological UICC tumour stage } \\
\hline & $\mathrm{n}=103$ & $\%$ \\
\hline Stage I & 34 & $33 \%$ \\
Stage IIa & 19 & $18 \%$ \\
Stage IIb & 5 & $5 \%$ \\
& & \\
Stage IIIa & 8 & $8 \%$ \\
Stage IIIb & 13 & $13 \%$ \\
Stage IIIc & 15 & $14 \%$ \\
Stage IV & 9 & $9 \%$ \\
\hline
\end{tabular}

Fifty percent $(51 / 103)$ of patients were staged to have advanced rectal cancer $(>\mathrm{T} 2$ or $\mathrm{N}+$ ). Of those, $61 \%(31 / 51)$ received neoadjuvant radiation, while $39 \%$ (20/51) underwent operation without preoperative radiation. The reasons for primary operation without radiation in advanced stages of rectal cancer included recurrence of rectal cancer $(7 \% ; 7 / 103)$, suspected metastatic disease $(9 \% ; 9 / 103)$, urgent surgery $(8 \%$; $8 / 103)$, advanced age $(2 \% ; 2 / 103)$, severe co-morbidities $(2 \% ; 2 / 103)$, incompliance $(1 \% ; 1 / 103)$, other malignancy $(1 \% ; 1 / 103)$ or combination of these.

The mean number of lymph nodes removed during the operation was 19 (range 4-47). Sixtysix percent of the removed tumours $(68 / 103)$ had moderate differentiation, $17 \%$ (18/103) good differentiation and $9 \%$ $(9 / 103)$ poor differentiation, while in $1 \%(1 / 103)$ an undifferentiated tumour was found. In $7 \%(7 / 103)$ of cases grading was not possible. Distant metastases were present in 9\% (9/103), including 6 patients with hepatic metastases, 1 patient with pulmonary and hepatic metastases and two patients with metastases towards other organs. In 91\% (94/103) patients had no distant metastases.

In $2 \%(2 / 103)$ of the cases a macroscopic residual tumour (R2) was present in the pelvis after resection. One of those patients had also a liver metastasis. In $98 \%(101 / 103)$ no residual tumour (R0) was left in situ. There was no case of microscopic residual tumour (R1). 5\% (5/103) of the patients had overall R2 resection because of hepatic or pulmonary metastasis, while $2 \%(2 / 103)$ of the patients underwent simultaneous hepatic tumour resection resulting in $\mathrm{R} 0$ situation.
Until May 2009 there has been one proven local recurrence. In November 2005 this patient had palliative rectal resection for a pT3b, pN2 (8/22), L1, V1, Pn1, pM1 (hepatic and pulmonary), G3, UICC-stage IV rectal cancer. The rectal cancer recurrence was diagnosed in May 2007.

\section{Discussion}

The prognosis of rectal cancer depends principally on tumour stage at the time of diagnosis, while local recurrence depends rather on surgical technique. In studies on colorectal cancer, survival is most important, but trials on rectal cancer also focus on local recurrence since local control also correlates with survival [10]. Conventional resections for rectal cancer result in recurrence rates between $20 \%$ and $40 \%$ [11]. Patients receiving preoperative radiotherapy before conventional resection for rectal cancer still have recurrence rates between $28 \%$ and $37 \%$ [12,13].

TME has been shown to result in reduced local recurrence and better overall survival [1]. The rate of local recurrence is directly related to the technique of rectal cancer excision, which makes surgical technique the most important factor for patients' outcome. Although TME is accepted in western countries as a standard principle of surgical treatment for rectal cancer the rate of local recurrence without radiotherapy varies between $3 \%$ and $18 \%[1,14]$.

Kapiteijn and his group could show that short term preoperative radiotherapy reduced the rate of local recurrence [6]. This analysis suggests that radiotherapy is the key for the reduction of local recurrence. But the fact that best results for rectal cancer surgery were reported without radiotherapy by Heald who originally introduced the principle of TME makes it obvious that the surgeon plays a crucial rule for patients ' prognosis. At a mean follow up of 4,2 years after curative resection for rectal cancer Heald reported a local recurrence rate of less than $3 \%$. Overall survival after 115 rectal resections was $87 \%$ [1].

Two questions arise:

1. Is the variation in local control in studies related to the surgical quality of TME?

2. How can a good TME quality be assured?

Although modern surgical treatment of colorectal cancer implies the operative principle of TME, data 
on TME quality of resected specimen are rare. The few analyses existing have shown a high rate of incomplete mesorectal excisions.

Nagtegaal et al. analysed TME quality among a subgroup of patients from the Dutch TME trial. They found that only $57 \%$ had undergone complete mesorectal excision (M.E.R.C.U.R.Y. $\mathrm{I}^{\circ}$ ). Local and distant recurrence rate was significantly higher after incomplete TME [7].

These results were confirmed by Maslekar et al. They analysed local and overall recurrence in accordance to the quality of TME surgery. TME quality was optimal in $47 \%, 40 \%$ of patients had nearly complete TME, while 13\% had incomplete TME. Local recurrence was related to the quality of surgery. In patients with incomplete TME the rate of local recurrence was $41 \%$ (59\% overall recurrence), in patients with nearly complete TME the rate of local recurrence was $6 \%$ (17\% overall recurrence), while in patients with optimal TME surgery the rate of local recurrence was less than $2 \%(2 \%$ overall recurrence) [15].

A study from Korea reported $75 \%$ optimal TME quality (M.E.R.C.U.R.Y. I ${ }^{\circ}$ ), but there were 12 patients with positive resection margins, which makes local recurrence likely to occur [16,17].

Hermanek and Heald also focused on the results of the rectal cancer study from the Netherlands. They showed that not the whole series of the Dutch trial did represent a standardized TME surgery since there was a high rate of incomplete mesorectal excisions for resectable rectal cancer [18]. The variation in local control therefore seems to be an indicator for differences in surgical quality of TME.

More evidence for the importance of surgical quality is the difference between multicentre trials and results from single institutions. Heald's excellent data were the result of a single person applying a new technique. The analysis from the Netherlands might have compaired excellent surgical quality with poorer quality from different institutions.

The role of the surgeon becomes even more obvious since objective analysis of the operative quality is possible by the evaluation of the quality of TME.

Therefore the documentation of TME quality by pathologists is essential to detect deficits in surgical technique. This may lead to better TME quality and seems to be an effective tool to improve operative results [19].

In our series we could demonstrate that optimal TME quality can be achieved with different operative procedures in any stage of rectal carcinoma. Although we had a relatively high rate of abdominoperineal resections this did not increase the rate of incomplete TME.

Other institutions showed that optimal TME quality can be achieved by individual training after instruction by TME trained surgeons [20]. All operations except for urgent surgery were supervised by surgeons being experienced in TME surgery for several years.

During the whole study operations were performed by 7 different surgeons from our institution. There were two cases of resection with residual tumour in final histology. Both patients underwent urgent surgery for a $\mathrm{T} 4$ rectal carcinoma.
The introduction of TME surgery can be associated initially with higher rates of anastomotic leakage, but several studies showed that these findings would improve during the routine application of TME surgery [21-23]. During the time of our study there were 4 of 78 cases with a clinical apparent anastomotic leakage. None of these patients needed a reoperation. This might be a result of the routine diverting ileostomy at our institution [24].

An explanation for the high rate of Hartmann`s stump leakage is that these were all urgent operations for perforated rectal cancer in patients requiring high dose catecholamines imparing wound healing. This fact may also be responsible for the high mortality rate after urgent surgery. Two patients died secondary to generalised peritonitis after Hartmann's operation, the other patient died due to excessive liver metastases after urgent colectomy.

In our series the mean number of removed lymph nodes was 19 (range 4-47). This result is within the range required for rectal cancer surgery [25]. Patients with less than 12 lymph nodes removed during the operation either had a local recurrence of rectal cancer or had undergone neoadjuvant radiation.

Apart from TME quality another marker for mastery in rectal cancer surgery is the achievement of negative resection margins. Inadequate surgical resection with lateral tumour spread will result in a local recurrence $[9,26]$. A distance of more than $1 \mathrm{~mm}$ from the tumour to the border of resection is considered to be a negative margin, although a recent analysis reported a distance of $2 \mathrm{~mm}$ to be the limit [17].

There may be further discussion about the exact distance for negative resections margins, but the optimal technique to obtain free resection margins is total mesorectal excision since it has been shown that TME achieves a negative resection margin in up to $96 \%$ of resected specimen [20]. These results were confirmed by Nagtegaal et al. They analysed the importance of TME to obtain free circumferential resection margins showing that in patients with positive CRM the rate of incomplete TME was $44 \%$ while in patients with negative CRM the rate of incomplete TME was only $11 \%$. Furthermore lateral margin involvement was more likely to occur in advanced tumour stages than in tumours with positive lymph nodes. In UICC stage III with positive CRM secondary to incomplete mesorectal excision there were significantly more patients with lateral margin involvement, by the primary tumour than by positive lymph nodes.

In our series the rate of positive circumferential margins was $2 \%$. Compared to the results from the literature ranging between $18 \%$ and $28 \%$ of resection margin involvement our findings reflect another effect of optimal TME surgery [7].

Taken both together, the accuracy of mesorectal excision and the analysis of circumferential resection margins are effective in predicting patients' prognosis.

Negative resection margins unfortunately do not only depend on surgical technique but on tumour size and tumour stage at the time of operation. Thus adequate staging prior to surgery is mandatory. Although radiotherapy has been shown to improve outcome for patients with resectable rectal cancer, subgroup analy- 
sis from the Dutch trial showed that there is no benefit for patients with UICC tumour stage I or IV in the upper part of the rectum [6]. Therefore it is important to improve selection criteria for the application of preoperative radiotherapy to protect patients from the side effects of radiation without benefit $[27,28]$. In our analysis we could show that optimal TME quality is feasible in all stages of rectal cancer.

\section{CONCLUSION}

High quality rectal cancer surgery implies the concept of total meserectal excision. The technique of TME can be learnt under supervision of specialised surgeons. High quality TME surgery can be performed with a rate of morbidity and mortality comparable to the results from the literature. With adequate surgical expertise high quality TME surgery is also feasible in advanced stages of rectal cancer. The application of a correct TME technique also results in free circumferential resection margins. Future studies should evaluate outcome and local recurrence in accordance to the degree of TME quality.

\section{LITERATURE}

1. Heald RJ, Ryall RD: Recurrence and survival after total mesorectal excision for rectal cancer. Lancet 1986;1:14791482.

2. Hill GL, Rafique M: Extrafascial excision of the rectum for rectal cancer. Br J Surg 1998;85:809-812.

3. Ross A, Rusnak C, Weinerman B, et al.: Recurrence and survival after surgical management of rectal cancer. Am J Surg 1999;177:392-395.

4. Bjerkeset T, Edna TH: Rectal cancer: the influence of type of operation on local recurrence and survival. Eur J Surg 1996;162:643-648.

5. M.E.R.C.U.R.Y.: (Magnetic Resonance Imaging and Rectal Cancer European Equivalence Study), Study Coordinator Daniels, I., Pelican Centre, North Hampshire Hospital, Basingstoke, Hampshire, UK, 2002. http://www.pelicancancer.org/researchprojects.

6. Kapiteijn E, Marijnen CA, Nagtegaal ID, et al.: Preoperative radiotherapy combined with total mesorectal excision for resectable rectal cancer. N Engl J Med 2001;345:638646.

7. Nagtegaal ID, van de Velde CJ, van der Worp E, et al.: Macroscopic evaluation of rectal cancer resection specimen: clinical significance of the pathologist in quality control. J Clin Oncol 2002;20:1729-1734.

8. Z'Graggen K, Maurer CA, Buchler MW: Transverse coloplasty pouch. A novel neorectal reservoir. Dig Surg 1999;16:363-366.

9. Quirke P, Durdey P, Dixon MF, Williams NS: Local recurrence of rectal adenocarcinoma due to inadequate surgical resection. Histopathological study of lateral tumour spread and surgical excision. Lancet 1986;2:996-999.

10. Improved survival with preoperative radiotherapy in resectable rectal cancer. Swedish Rectal Cancer Trial. N Engl J Med 1997;336:980-987.

11. Hermanek P, Wiebelt H, Staimmer D, Riedl S: Prognostic factors of rectum carcinoma--experience of the German Multicentre Study SGCRC. German Study Group ColoRectal Carcinoma. Tumori 1995;81:60-64.

12. Gerard A, Buyse M, Nordlinger B, et al.: Preoperative radiotherapy as adjuvant treatment in rectal cancer. Final results of a randomized study of the European Organization for Research and Treatment of Cancer (EORTC). Ann Surg 1988;208:606-614.
13. Roswit B, Higgins GA, Keehn RJ: Preoperative irradiation for carcinoma of the rectum and rectosigmoid colon: reportof a National Veterans Administration randomized study. Cancer 1975;35:1597-1602.

14. Hainsworth PJ, Egan MJ, Cunliffe WJ: Evaluation of a policy of total mesorectal excision for rectal and rectosigmoid cancers. Br J Surg 1997;84:652-656.

15. Maslekar S, Sharma A, Macdonald A, et al.: Mesorectal grades predict recurrences after curative resection for rectal cancer. Dis Colon Rectum 2007;50:168-175.

16. Hyuk Baik S, Kyu Kim N, Young Lee K, et al.: Factors Influencing Pathologic Results after Total Mesorectal Excision for Rectal Cancer: Analysis of Consecutive 100 Cases. Ann Surg Oncol 2007;5:5.

17. Nagtegaal ID, Marijnen CA, Kranenbarg EK, et al.: Circumferential margin involvement is still an important predictor of local recurrence in rectal carcinoma: not one millimeter but two millimeters is the limit. Am J Surg Pathol 2002;26:350-357.

18. Hermanek P, Heald RJ: Pre- operative radiotherapy for rectal carcinoma? Has the case really been made for shortcourse pre- operative radiotherapy if surgical standards for rectal carcinoma are optimal? Colorectal Dis 2004;6:10-14.

19. Hermanek P, Hohenberger W, Klimpfinger M, et al.: The pathological assessment of mesorectal excision: implications for further treatment and quality management. Int J Colorectal Dis 2003;18:335-341.

20. Martling AL, Holm T, Rutqvist LE, et al.: Effect of a surgical training programme on outcome of rectal cancer in the County of Stockholm. Stockholm Colorectal Cancer Study Group, Basingstoke Bowel Cancer Research Project. Lancet 2000;356:93-96.

21. Carlsen E, Schlichting E, Guldvog I, et al.: Effect of the introduction of total mesorectal excision for the treatment of rectal cancer. Br J Surg 1998;85:526-529.

22. Karanjia ND, Corder AP, Bearn P, Heald RJ: Leakage from stapled low anastomosis after total mesorectal excision for carcinoma of the rectum. Br J Surg 1994;81: 1224-1226.

23. Alves A, Panis Y, Mathieu P, et al.: Mortality and morbidity after surgery of mid and low rectal cancer. Results of a French prospective multicentric study. Gastroenterol Clin Biol 2005;29:509-514.

24. Karanjia ND, Corder AP, Holdsworth PJ, Heald RJ: Risk of peritonitis and fatal septicaemia and the need to defunction the low anastomosis. Br J Surg 1991;78:196-198.

25. Edler D, Ohrling K, Hallstrom M, et al.: The number of analyzed lymph nodes - a prognostic factor in colorectal cancer. Acta Oncol 2007;46:975-981.

26. Adam IJ, Mohamdee MO, Martin IG, et al.: Role of circumferential margin involvement in the local recurrence of rectal cancer. Lancet 1994;344:707-711.

27. Peeters KC, van de Velde CJ, Leer JW, et al.: Late side effects of short-course preoperative radiotherapy combined with total mesorectal excision for rectal cancer: increased bowel dysfunction in irradiated patients--a Dutch colorectal cancer group study. J Clin Oncol 2005;23:6199-6206.

28. Marijnen CA, van de Velde CJ, Putter H, et al.: Impact of short-term preoperative radiotherapy on health-related quality of life and sexual functioning in primary rectal cancer: report of a multicenter randomized trial. J Clin Oncol 2005;23:1847-1858.

Received: August 31, 2009 / Accepted: November 2, 2009

Address for correspondence:

Matthias H. Seelig, MD

Department of Surgery

St. Josef Hospital Bochum,

Gudrunstr. 56

44791 Bochum, Germany

Phone: +49234 5092210

Fax: +492345092209

E-mail: m.seelig@klinikum-bochum.de 